\title{
Spectra and fast multi-wavelength variability of compact jets powered by internal shocks
}

\author{
Julien Malzac $^{1,2}$ and Samia Drappeau ${ }^{1,2}$ \\ ${ }^{1}$ Université de Toulouse; UPS-OMP, IRAP, Toulouse, France \\ ${ }^{2}$ CNRS, IRAP, 9 Av. colonel Roche, BP 44346, F-31028 Toulouse cedex 4, France \\ email: julien.malzac@irap.omp.eu
}

\begin{abstract}
The emission of steady compact jets observed in the hard spectral state of X-ray binaries is likely to be powered by internal shocks caused by fluctuations of the outflow velocity. The dynamics of the internal shocks and the resulting spectral energy distribution (SED) of the jet is very sensitive to the shape of the Power Spectral Density (PSD) of the fluctuations of the jet Lorentz factor. It turns out that Lorentz factor fluctuations injected at the base of the jet with a flicker noise power spectrum (i.e. $P(f) \propto 1 / f$ ) naturally produce the canonical flat SED observed from radio to IR band in X-ray binary systems in the hard state. This model also predicts a strong, wavelength dependent, variability that resembles the observed one. In particular, strong sub-second variability is predicted in the infrared and optical bands. The assumed fluctuations of the jet Lorentz factor are likely to be triggered by the variability of the accretion flow which is best traced by the X-ray emission. In the case of GX339-4 for which high quality and simultaneous multi-wavelength data are available, we performed simulations assuming that the fluctuation of the jet Lorentz factor have the same PSD as the observed X-ray PSD. The synthetic SED calculated under this assumption provides a remarkable match to the observed radio to IR SED. In this case the model also produces strong mid-infrared spectral variability that is comparable to that reported in this source.
\end{abstract}

Keywords. accretion, accretion discs, black hole physics, shock waves, relativistic processes, radiation mechanisms: non-thermal, X-rays: binaries

\section{Properties of the internal shock model}

Steady compact jets are probably the most common form of jets in X-ray binaries. They appear to be present in all black hole and neutron star binaries when in the hard X-ray spectral state. They have an approximatively flat Spectral Energy Distribution (SED) extending from the radio to the mid-IR (e.g. Fender et al. 2000; Corbel \& Fender 2002; Chaty et al. 2003; Migliari et al. 2011). These flat spectra are usually ascribed to self-absorbed synchrotron emission from conical compact jets (Blandford \& Königl 1979) under the assumption of continuous energy replenishment of the adiabatic losses. The compensation of these energy losses is crucial for maintaining this specific spectral shape (Kaiser 2006).

Internal shocks provide a possible mechanism to compensate the adiabatic losses by dissipating energy and accelerating particles at large distance from the black hole. Internal shocks caused by fluctuations of the outflow velocity are indeed widely believed to power the multi-wavelength emission of jetted sources such as $\gamma$-ray bursts (Rees \& Meszaros 1994; Daigne \& Moscovitch 1998), active galactic nuclei (Rees 1978; Spada et al. 2001), or microquasars (Kaiser, Sunyaev \& Spruit 2000; Jamil et al. 2010). Internal shocks models usually assume that the jet can be discretised into homogeneous ejecta. Those ejecta are injected at the base of the jet with variable velocities and then propagate along the jet. At some point, the fastest fluctuations start catching up and merging with slower 



Figure 1. Simulation of the internal shock model with a power-law PSD of the Lorentz factor fluctuations $\left(P(f) \propto f^{-\alpha}\right)$. The top left panel shows the shape of the injected PSDs, for the indicated values of the $\alpha$ index. The right panel shows the jet SED calculated for an inclination angle of 40 degrees and a distance to the source of 2 Kpc. See Malzac (2014) for details.

ones. This leads to shocks in which a fraction of the bulk kinetic velocity of the shells is converted into internal energy. Part of the dissipated energy goes into particles acceleration, leading to synchrotron and also, possibly, inverse Compton emission. Recently, however, Jamil et al. (2010) developed an internal shock model for the emission of jets in $\mathrm{X}$-ray binaries, and concluded that energy dissipation through internal shocks only is not enough to produce a flat SED. Nevertheless, most studies of the internal shock model so far, including that of Jamil et al. (2010), have implicitly assumed that the Fourier Power Spectral Density (PSD) of the velocity fluctuations injected at the base of the jet is flat (i.e. white noise). In fact, the energy dissipation profile of the internal shocks is very sensitive to the shape of the PSD of the velocity fluctuations. Indeed, let us consider a fluctuation of the jet velocity of amplitude $\Delta v$ occurring on a time scale $\Delta t$. This leads to the formation of a shock at a downstream distance $z_{s} \propto \Delta t / \Delta v$. In this shock the fraction of the kinetic energy converted into internal energy will be larger for larger $\Delta v$. From these simple considerations we see that the distribution of the velocity fluctuation amplitudes over their time scales (i.e. the PSD) is going to determine where and in which amount the energy of the internal shocks is deposited. Malzac (2014) used Monte-Carlo simulations to study this dependance and found that independently of the details of the model flat radio-IR SEDs are obtained for a flicker noise PSD of the fluctuations of the jet Lorentz factor. This result is illustrated by Fig. 1, which compares the SEDs obtained for PSD of the Lorentz factor of the jet with a power-law shape with varying index $\alpha$ : $P(f) \propto f^{-\alpha}$. For larger $\alpha$ the fluctuations of the Lorentz factor have, on average, longer time-scales and therefore more dissipation occurs at larger distances from the black hole. One can see from Fig. 1 that the SED is very sensitive to the value of $\alpha$, for $\alpha=1$ (i.e. flicker noise) the dissipation profile scales like $z^{-1}$ and the specific energy profile is flat. In other words, the internal shocks compensate exactly for the adiabatic losses. As result the SED is flat over a wide range of photon frequencies. In fact, this result can also be obtained analytically (Malzac 2013). The case of flicker noise fluctuations of the jet Lorentz factor may therefore be relevant to the observations of compact jets.

An interesting feature of the internal shock model is that it naturally predicts strong variability of the jet emission. Figure 2 shows sample light curves and power spectra obtained from the simulation with $\alpha=1$. The jet behaves like a low-pass filter. As the shells of plasma travel down the jet, colliding and merging with each other, the highest frequency velocity fluctuations are gradually damped and the size of the emitting region increases. The jet is strongly variable in the optical and IR bands originating 

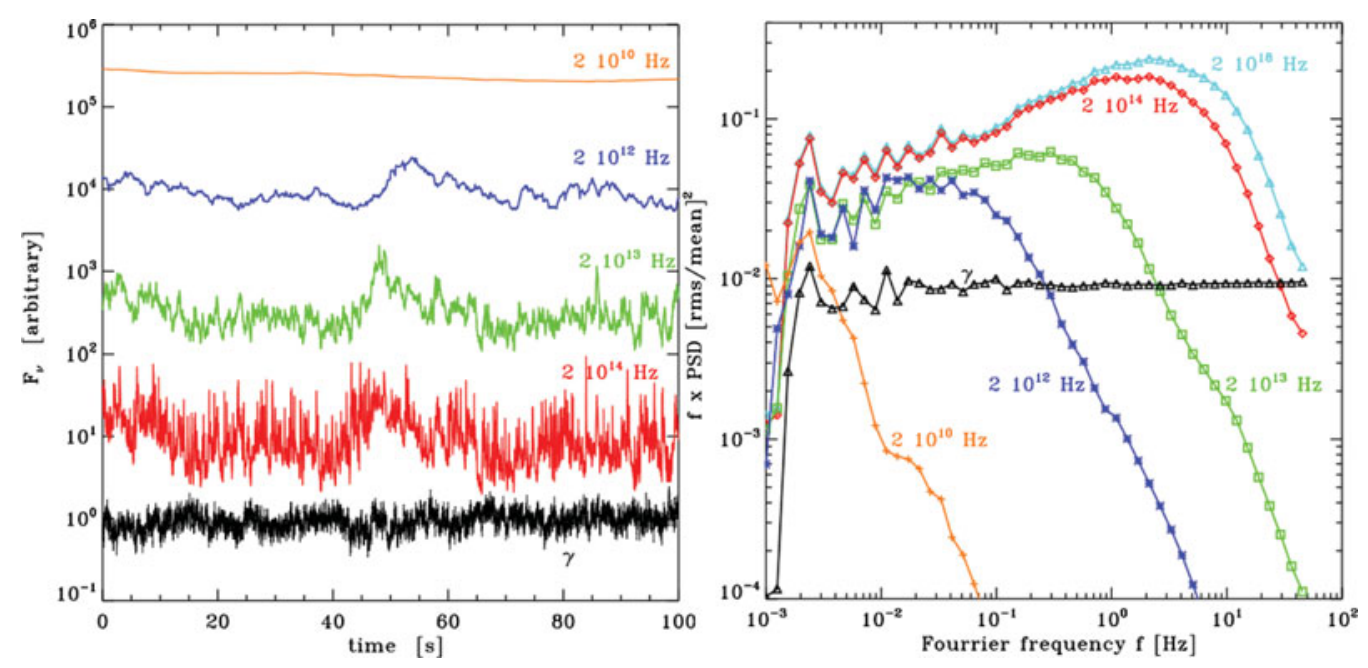

Figure 2. Synthetic light curves (left, rescaled) and power spectra at various indicated frequencies resulting from the simulation with $\alpha=0$. The injected fluctuations of the Lorentz factor are also shown.

primarily from the base of the emitting region and becomes less and less variable at longer frequencies produced at larger distances from the black hole. The observations also show significant flickering in the Infrared and optical band (Kanbach et al. 2001; Casella et al. 2010; Gandhi et al. 2010). At least part of this fast IR/optical variability is likely to arise from the jet, possibly through internal shocks. Another interesting property of the observed variability is the existence of correlations with the fast X-ray variability originating from the accretion flow. In particular, Casella et al. (2010) measured de crosscorrelation function of the X-ray and IR light curves and found significant correlation between the two bands with the infrared photons lagging behind the X-rays by about $100 \mathrm{~ms}$. Casella et al. (2010) interpreted this time-lag as the propagation time of the ejected shells from the accretion flow to the infrared emitting region in the jet. In the framework of the internal shock model this observation suggests that the fluctuations of the jet Lorentz factor are related to the X-ray variability of the source.

\section{Are the jet Lorentz factor fluctuations related to the X-ray variability?}

In fact, if the jet is launched from the accretion disc, the variability of the jet Lorentz factor must be related to that of the accretion disc. And we know, both from theory (see e.g., Lyubarskii 1997) and from observations (see e.g. Gilfanov \& Arefiev 2005) that accretion discs tend to generate flicker noise variability. Therefore flicker noise fluctuations of the jet Lorentz factor are not unexpected. In X-ray binaries the variability of the accretion flow is best traced directly by the X-ray light curves. Those sources indeed exhibit a strong variability over a very broad range of time scales. This is consistent with the idea that the fluctuations of the jet Lorentz factor may be related to the X-ray variability. Although this variability is close to flicker noise, it appears to be more complex. The left panel of Fig. 3) shows an actual X-ray PSD of the black hole binary GX339-4. As can be seen on this figure, in the hard state, which is the spectral state associated to the presence of compact radio jets, the short time-scale X-ray variability is dominated by 



Figure 3. Left: the observed X-ray PSD of GX 339-4 during the observations presented in Gandhi et al. (2011). Right: the SED measured by Gandhi et al. (2011) compared to a simulated jet SED obtained assuming that the jet Lorentz factor fluctuations have exactly the PSD as the X-ray flux.

a band limited noise that is well described in terms of 4-5 broad Lorentzians (e.g. Nowak 2000; van der Klis 2006).

This variability could be a good proxy for the assumed fluctuations of the jet. The right panel of Fig. 3 shows the resulting time averaged SED obtained if one assume that the Lorentz factor fluctuations have a PSD that is exactly the observed X-rays. This synthetic SED is compared to multi wavelength observation that are nearly simultaneous with the X-ray timing data (see Gandhi et al. 2011 for details). The model appears to reproduce pretty well the radio to infrared data. This agreement is striking because the shape of the SED depends almost uniquely on the assumed shape of the PSD of the fluctuations. Although the model has a number of free parameters (jet power, inclination angle, timeaveraged jet Lorentz factor...) that could be tuned to fit the data, those parameters only allow to modify the flux normalisation or shift it in the photon frequency direction (Drappeau et al. submitted), but they have very little effects on the overall shape of the SED.

The four mid-infrared flux measurements at $1.36 \times 10^{13}, 2.50 \times 10^{13}, 6.52 \times 10^{13}$ and $8.82 \times 10^{13} \mathrm{~Hz}$ that are shown on Fig. 3 were obtained with the Wide field Infrared Survey Explorer (WISE; Wright et al. 2010) they represent an average over 13 epochs, sampled at multiples of the satellite orbital period of 95 minutes and with a shortest sampling interval of $11 \mathrm{~s}$, when WISE caught the source on two consecutive scans. These data have revealed a strong variability of the mid-infrared emission (see Gandhi et al. 2011). The light curves of these observations are shown in Fig. 4 (left panel) and compared to light curves obtained from the same simulation that gives a good fit to the observed radio-IR SED (right panel). The model appears to predict a variability of similar amplitude to that observed by WISE.

\section{Conclusion}

Internal shocks naturally lead to the formation of the observed SEDs of compact jets and also predict a strong, wavelength dependent, variability that resembles the observed one. The assumed velocity fluctuations of the jet must originate in the accretion flow. The model thus predicts a strong connection between the observable properties of the jet in the radio to IR bands, and the variability of the accretion flow as observed in X-rays. If the model is correct, this offers a unique possibility to probe the dynamics of the coupled accretion and ejection processes leading to the formation of compact jets. 

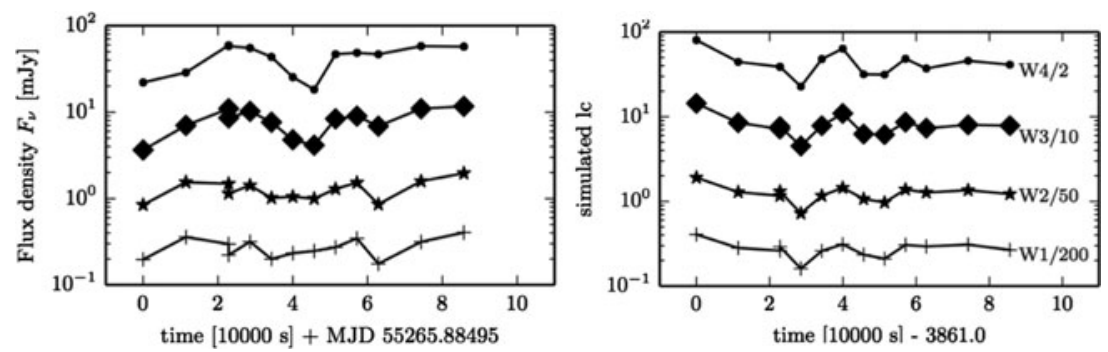

Figure 4. Left: the observed mid-infrared variability as observed by WISE in 4 bands. Right: sample synthetic light curves obtained from the same simulation shown in Fig. 3.

\section{References}

Blandford, R. D. \& Königl, A., 1979, ApJ, 232, 34

Casella, P., et al., 2010, MNRAS, 404, L21

Chaty, S., et al., 2003, MNRAS, 346, 689

Corbel, S. \& Fender, R. P., 2002, ApJ, 573, L35

Daigne, F. \& Mochkovitch, R., 1998, MNRAS, 296, 275

Drappeau, S., Malzac, J., Belmont, R., Gandhi, P., \& Corbel, S., 2015, MNRAS, 447, 3832

Fender, R. P., et al., 2000, MNRAS, 312, 853

Gandhi, P., et al., 2010, MNRAS, 407, 2166

Gandhi, P., et al., 2011, ApJ, 740, L13

Gilfanov, M. \& Arefiev, V., 2005, arXiv:astro-ph/0501215

Jamil, O., Fender, R. P., \& Kaiser, C. R., 2010, MNRAS, 401, 394

Kaiser, C. R., Sunyaev, R., \& Spruit, H. C., 2000, A\&A, 356, 975

Kaiser, C. R., 2006, MNRAS, 367, 1083

Kanbach, G., Straubmeier, C., Spruit, H. C., \& Belloni, T., 2001, Nature, 414, 180

Lyubarskii, Y. E., 1997, MNRAS, 292, 679

Malzac, J., 2013, MNRAS, 429, L20

Malzac, J., 2014, MNRAS, 443, 299

Migliari, S., Miller-Jones, J. C. A., \& Russell, D. M., 2011, MNRAS, 415, 2407

Nowak, M. A., 2000, MNRAS, 318, 361

Rees, M. J., 1978, MNRAS, 184, 61P

Rees, M. J. \& Meszaros, P., 1994, ApJ, 430, L93

Spada, M., Ghisellini, G., Lazzati, D., \& Celotti, A., 2001, MNRAS, 325, 1559

van der Klis, M., 2006, in Compact stellar X-ray sources, Lewin \& van der Klis (eds), Cambridge University Press, arXiv:astro-ph/0410551

Wright, N. J., Drake, J. J., \& Civano, F., 2010, ApJ, 725, 480 